\title{
Announcement: PRFluids publishes Invited Perspective on Machine Learning for Advancing Fluid Mechanics
}

The application of machine learning methods to advance the science of fluid mechanics is emerging as an important research area. A symposium on machine learning in fluid mechanics will be held at the upcoming 2019 APS Division of Fluid Dynamics conference.

As a prologue to the symposium and to encourage future papers in this area Physical Review Fluids has published the invited paper "Perspective on machine learning for advancing fluid mechanics" which describes recent developments in machine learning and how they may advance fluid mechanics. Current limitations are discussed, though the potential impact is deemed high, so long as outcomes are held to the long-standing critical standards that should guide studies of flow physics.

Published 16 October 2019

DOI: 10.1103/PhysRevFluids.4.100001 